\title{
ÍNDICE DE SITIO (IS) DE Calycophyllum spruceanum Benth. EN RELACIÓN CON LA ALTURA DOMINANTE DEL RODAL EN ENSAYOS DE PLANTACIÓN EN LA CUENCA DEL AGUAYTÍA, UCAYALI, PERÚ
}

\section{SITE INDEX OF Calycophyllum spruceanum Benth. IN RELATION WITH THE DOMINANT HEIGHT OF ESSAY PLANTATIONS IN THE AGUAYTÍA BASIN, UCAYALI, PERU}

\author{
Leoncio Julio Ugarte-Guerra ${ }^{1}$ y Gilberto Domínguez-Torrejón ${ }^{2}$
}

\begin{abstract}
Resumen
El objetivo fue evaluar el efecto del sitio en la productividad de 12 plantaciones (repeticiones) de Calycophyllum spruceanum Benth. con una extensión total de 5.72 ha Estas plantaciones se distribuyen en 3 bloques coincidentes con la parte alta, media y baja de la cuenca del río Aguaytía, Región Ucayali, Perú. Se aplicó el método dasométrico para conocer el estado actual de las plantaciones y su relación con las variables edáficas y de precipitación para identificar los potenciales sitios para plantaciones exitosas de Calycophyllum spruceanum Benth. en la zona. Se calculó y definió el Î́ndice de Sitio como el promedio de altura en metros del quintíl más alto. El Índice de Sitio presentó una alta correlación con el área basal y el volumen maderable total.

Se encontraron diferencias notables en las parcelas evaluadas por lo que el estudio permite generar una clasificación sobre la base del índice de sitio. Esta clasificación coincide con la agrupación por bloques y la altura de la cuenca en la mayoría de los casos. El crecimiento en el mejor bloque dio como resultado un incremento volumétrico de hasta $13.349 \mathrm{~m}^{3} \mathrm{ha}^{-1}$ año-1.

Palabras clave: Silvicultura, plantaciones forestales, índice de sitio, productividad, Calycophyllum spruceanum, Ucayali, Perú.
\end{abstract}

\begin{abstract}
The objective was to evaluate the effect of the site in the productivity of 12 Calycophyllum spruceanum Benth. forest plantations (replications) in a total area of 5.72 has. Plantations were distributed into 3 blocks in the Aguaytía River, located in the Ucayali Region. Direct measurements were used to evaluate the current state of the plantations and their relationship with soil and climate factors in order to identify potential places for the establishment of successful plantations. Site Index (SI), was defined as the average of the total height in meters of the higher quintile. Site Index was highly correlated to basal area, and total bark volume.

Noteworthy differences were found in the plots evaluated. This allows the generation of a classification based in the Site Index. This classification is consistent with the blocks and the altitude throughout the basin in most of the cases. Growth average in the best replication resulted in a volumetric increment of up to $13.349 \mathrm{~m}^{3} \mathrm{ha}^{-1} \mathrm{year}^{-1}$.

Key words: Silviculture, forest plantations, Site Index, Productivity, Calycophyllum spruceanum, Ucayali, Perú.
\end{abstract}

\section{Introducción.}

Parte de los árboles aprovechados de Calycophyllum spruceanum Benth. en la región de Ucayali provienen de la regeneración natural que se establece en terrenos de barbecho. Esta especie al igual que otras pioneras de creciente aceptación en las últimas décadas, se han favorecido del proceso de extracción maderera en bosques primarios y la agricultura migratoria que la sigue. En la apertura de áreas para cultivo, muchas veces los árboles padres han dispersado sus semillas sobre terrenos recién abandonados o al inicio de la sucesión.
Las evaluaciones de Calycophyllum spruceanum Benth. han dado muestras de tener ritmos de crecimiento aceptables en suelos de fertilidad relativamente baja (Sotelo \& Weber, 2001). Teniendo en cuenta la densidad media-alta de la madera, la buena durabilidad natural, la aceptación en el mercado y la amplia distribución en la amazonía peruana de Calycophyllum spruceanum Benth, esta especie presenta gran potencial para plantaciones. En comparación a otras que crecen sobre terrenos de barbecho, esta especie tiene además demanda de productos de dimensiones mayores como madera estructural y madera para muebles por lo que plantaciones requieren incrementar la eficiencia de 
uso de la tierra mediante la mejora de los conocimientos sobre sus requerimientos ambientales y de suelos.

Los estudios para la evaluación del potencial del sitio en plantaciones con especies nativas maderables de la amazonía peruana son escasos, sin embargo existente una evaluación precedente para Caderelinga cateniformis (Tornillo) para esta zona elaborada por Flores (2008).

A lo largo del ciclo biológico del árbol el crecimiento en altura es determinado por la combinación de los factores potenciales (como la interacción entre las características genéticas y de sitio) y los factores limitantes del crecimiento siendo el principal la competencia por los recursos. (Chapman \& Myers, 1949; Froese et al., 2002). Las tasas de crecimiento en altura son menores en estadios iniciales, se incrementan en árboles jóvenes y tienden a estabilizarse en individuos adultos (Zedaker et al., 1987; Armitage \& Burley, 1980). Los modelos de crecimiento en altura se usan comúnmente por su buen ajuste a los modelos de crecimiento (Lencar \& Marshall, 2000; Wykoff, 1990; García 1999; McNab \& Ritter, 1999). Las combinaciones entre factores edáficos y climáticos son suficientemente precisas para determinar productividad promedio a nivel regional para algunas especies de clima templado (Tyler et al., 1996).

La productividad forestal (definida como la cantidad de materia producida por un área forestal por unidad de insumo) es un concepto biológico y difícilmente puede ser expresada matemáticamente, por ello se ha optado por representar la calidad del sitio mediante estimaciones indirectas (Thrower, 1989; Prodan et al., 1997; Casaubon et al., 2000) a través de un valor o índice denominado índice de sitio y/o índice de productividad (Prodan et al. 1997). Índice de sitio es la medida del potencial de la productividad forestal (Sharma et al., 2001; Canadian Forest Services, 2002), prediciendo no sólo la productividad sino también, el volumen de madera y la tasa de crecimiento potencial de un bosque (Hanson et al., 2002). Este concepto se ha desarrollado, para simplificar el proceso de evaluación y clasificación de rodales, y a pesar que (aun hoy en día) se trata de desarrollar un índice más evolucionado, ninguna otra aproximación es tan práctica, consistente y se encuentra tan ampliamente difundida como el índice de sitio (Baskaran, 2001; Avery \& Bukhart, 1994; Caillez y Alder, 1980; Beck, 1971; Jones, 1969). Se reconoce que no es un parámetro perfecto, pero en compensación es rápido y fácil de medir, de conceptuar y actualmente es la forma mas común de determinar la productividad de un área determinada (Sims, 1994; Avery \& Bukhart, 1994; Huebschmann \& Martin, 1996; Prodan et al., 1997; Hanson et al., 2002; Sharma et al., 2001; Parker et al., 2003). Se define al índice de sitio como la altura a la que puede crecer los árboles en condiciones naturales en un tiempo determinado (Daniels et al., 1979). Debido a que los rodales pueden ser muy heterogéneos, se prefieren las estimaciones basadas en la altura promedio de los árboles sanos dominantes y codominantes, que han crecido libremente, sin daños físicos ni mecánicos a una edad determinada denominada edad índice (Carmean, 1971; Prodan et al, 1997; Payandeh \& Wang, 1984 citado por Torres Rojo, 2001; Hanson et al., 2002; Canadian Forest Services, 2002) , o los árboles más gruesos (en caso de no tener la edad) a densidades medias o altas (Chapman \& Myers, 1949; Avery \& Bukhart, 1994). El índice de sitio alcanza una buena aproximación cuando:

(a) Los árboles adecuados para formular el índice son ubicados fácilmente en la edad y altura requerida y

(b) Se ha construido una curva específica para la calidad del sitio de precisión adecuada (Sims, 1994) variando de acuerdo a la especie, la zona geográfica y las condiciones de suelo (Huebschmann \& Martín, 1996).

El objetivo del presente artículo es evaluar las características fisiográficas, edáficas y climáticas que tiene mayor influencia en el crecimiento de la especie, basado en el índice de sitio (IS) en relación con la altura dominante.

\section{Materiales y métodos. \\ Características del área de estudio.}

Las parcelas de evaluación se ubican en la cuenca del río Aguaytía, Provincias de Padre Abad y Coronel Portillo, Región de Ucayali Perú (Fig. 1). El área de estudio esta conformada por la cuenca del Río Aguaytía, tributario del Río Ucayali y cubre una superficie de 1762086 ha con una población de 360 000 habitantes. La cuenca del Aguaytía se extiende hacia el sudoeste de la ciudad de Pucallpa, presenta un paisaje montañoso del lado occidental mientras que hacia el este es predominante plano e inundable. Por la facilidad de acceso terrestre y la promoción de políticas de colonización, la población ha crecido rápidamente y la deforestación se ha concentrado a ambos lados de la carretera Federico Basadre (CFB) y las carreteras secundarias a esta vía principal.

Plantaciones evaluadas.

Las plantaciones fueron establecidas con semillas colectada en junio de 1998 en el ámbito de la cuenca del Aguaytía. La produccion se inicio en julio de 1999 y las plantas alcanzaron $40 \mathrm{~cm}$ de altura en diciembre del 2000. Paralelamente se llevó a cabo labores de acondicionamiento de las áreas para plantación. Las plántulas fueron establecidas entre febrero y abril del 2001 dentro de la zona no inundable de la cuenca del río Aguaytía. Todas las repeticiones poseen un borde no evaluable formado por al menos dos árboles de alrededor de la repetición, adicionalmente se rodea 
con una faja corta fuego de $5 \mathrm{~m}$ de ancho y un cerco de alambre de puas. Después de los primeros 3 meses de crecimiento se sembró Centrosema macroporum, leguminosa que ayudaría a mantener el avance de plantas invasoras y ayudaría a la mantención de una cubierta sobre el suelo y disminuiría la erosión. En el momento de la plantación se aplicó 1600 kg de humus de lombriz ha-1 y $320 \mathrm{~kg}$ de roca fosfórica $\mathrm{ha}^{-1}$ (1 kg de humus de lombris y 200 g de roca fosfórica por planta a un distanciamiento de $2.5 \mathrm{~m} \mathrm{x}$ $2.5 \mathrm{~m}$.). Durante el segundo y tercer año se repetieron las fertilizaciones 112 $\mathrm{kg}$ de urea $\mathrm{ha}^{-1}, 316 \mathrm{~kg}$ roca fosfórica $\mathrm{ha}^{-1}$ y $160 \mathrm{~kg} \mathrm{ha}^{-1}$ de cloruro de potasio (correspondiendo respectivamente a 70

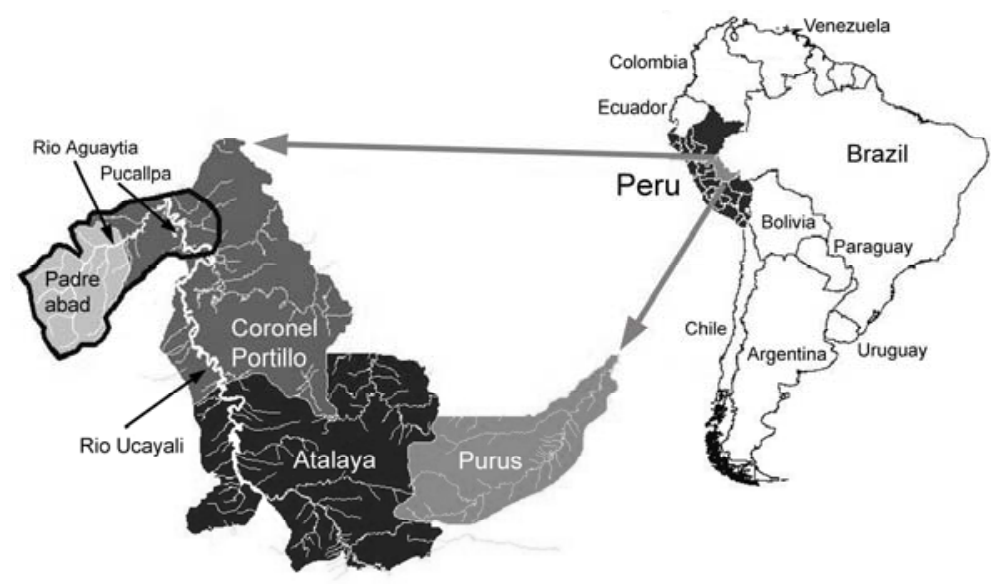
g, 185 g, y 100 g de cada producto por planta). Todas las repeticiones fueron establecidas participamente en parcelas de agricultores los que se comprometieron a dar mantenimiento en coordinación y bajo supervisión técnica del equipo de ICRAF, sin embargo, no ha sido posible asegurar el mismo nivel de mantenimiento para todas las repeticiones. Se recalzaron las repeticiones hasta en tres ocasiones durante el primer año de establecimiento para mantener la densidad inicial. En la evaluación de los 64 meses solo se consideró 12 repeticiones para el análisis debido a que 3 de las 5 repeticiones de la parte baja de la cuenca habían sido perdidas por acción del fuego a los 36 meses de edad.

Precipitación.

De acuerdo a la clasificación de zonas de vida (Holdridge, 1979) todas las plantaciones evaluadas (repeticiones en el diseño experimental) se encuentran en el denominado bosque premontano tropical y existe una relación con el mapa de precipitación de la cuenca del Aguaytía, Región Ucayali, Perú, construido en base a los datos de la base de datos meteorológica World clim (Hijmans et al., 2005) que permite hacer isoyetas basadas en datos de precipitación. En esta se presenta una marcada tendencia al aumento de la precipitación total desde la zona baja que es la menos húmeda hacia las partes más altas, siendo la parte más húmeda de la cuenca la zona cercana a Aguaytía, muy cerca de San Alejandro, que es la zona donde se ubica el bloque 3. Del mismo modo de acuerdo a los diagramas climáticos (Anexo 2) las partes bajas correspondiente al bloque 1 presenta un periodo de déficit hídrico de 5 meses, el bloque 2 de 4 meses y el bloque 3 de 1 mes. Este hecho indica una obvia ventaja de crecimiento para las partes altas.

La cuenca se ubica en el lado más occidental de la selva baja, pertenece a la región de Ucayali y en ella se encuentran diversas zonas de vida (Holdridge, 1979) predominando el bosque premontano tropical.

La cuenca se ubica entre las UTM 400000 y 600000 Oeste y 8960000 y 9120000 Sur. Los datos de temperatura son variables de acuerdo a la elevación siendo la temperatura anual media $26^{\circ} \mathrm{C}$.

Los suelos en la parte alta de la cuenca son ligeramente ácidos $(\mathrm{pH}$ 6.0) con niveles altos de disponibilidad de fósforo, potasio, calcio y magnesio. La textura del suelo es muy arenosa en la parte baja, con más arcilla en la parte media y ligera cantidad de materia orgánica en la parte alta de la cuenca. Los suelos en la parte más baja y media de la cuenca son muy ácidos (20-50 \% saturación de aluminio), con niveles bajos de disponibilidad de fósforo, y niveles intermedios de potasio, calcio y magnesio. Es necesario notar que en las áreas cercanas al cauce del río Aguaytía existen suelos de origen aluvial que son muy fértiles y de textura arcillosa o arcillo limosa.

Topografía y relieve.

Las parcelas del presente estudio están distribuidas sobre un área que abarca $100 \mathrm{~km}$ de E-O y $60 \mathrm{~km}$ de N-S. El perfil altitudinal de la cuenca está conformado por un paisaje ondulado en la parte más alta, que es propio de la transición entre los andes y la llanura amazónica y un paisaje plano en la parte baja, propio de la llanura amazónica. La elevación desciende de aproximadamente 300 a $180 \mathrm{~m}$. Los procesos tectónicos, geológicos y geomorfológicos, y los constantes cambios climáticos han originado la configuración actual del relieve.

Unidades experimentales.

Se han evaluado 12 rodales de Calycophyllum spruceanum Benth. de 64 meses de edad distribuidos en la cuenca del río Aguaytía, Provincia de Coronel Portillo Ucayali Perú con una extensión total de 5.72 ha (Figura 2).

Diseño experimental.

El diseño original del experimento fue de 15 repeticiones de bloques completos (15 repeticiones de 0.25 ha de extensión por repetición) los cuales por la pérdida de unidades experimentales se convirtieron en 


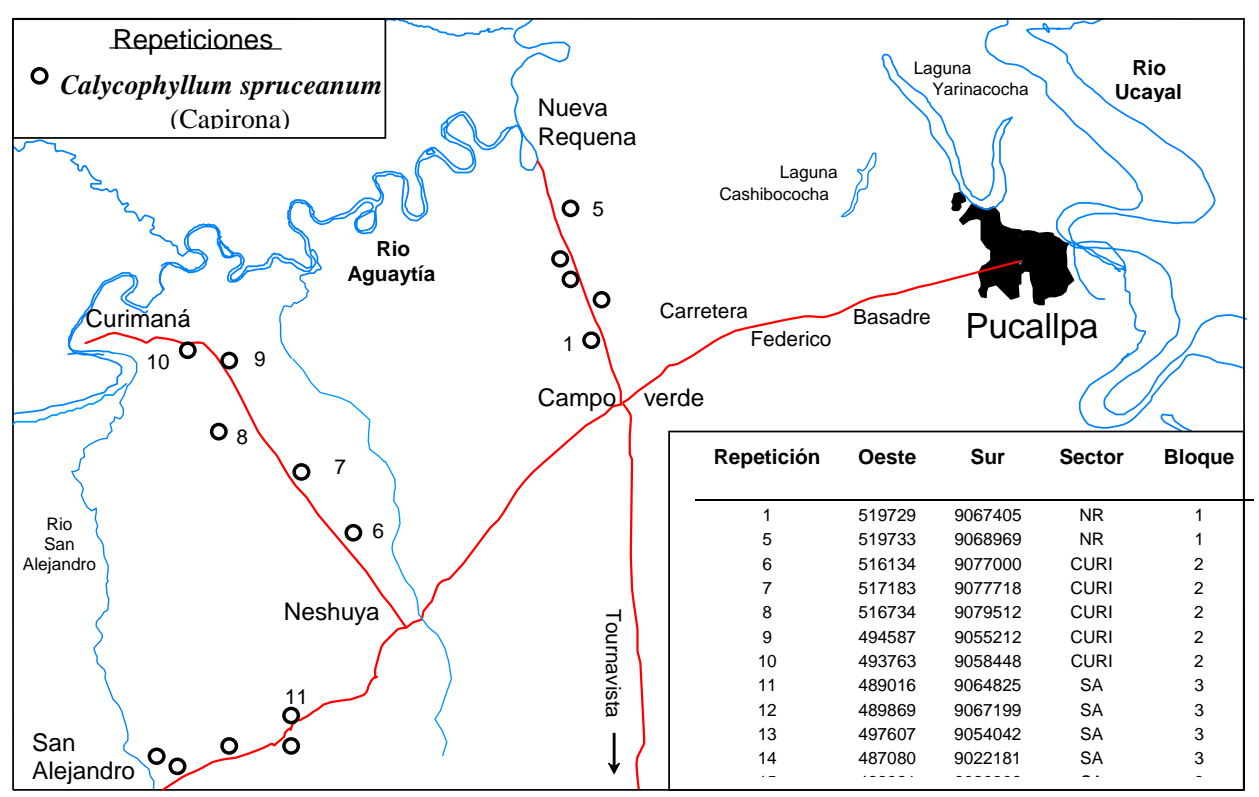

Figura 2. Ubicación de las parcelas evaluadas de Calycophyllum spruceanum Benth.
Cálculo del índice de sitio.

El Índice de Sitio estimado en base al promedio de las alturas de los árboles del quintil más alto que han sido dominantes $\mathrm{y} / \mathrm{o}$ co-dominantes en mediciones sucesivas ha sido un parámetro de fácil cálculo y alta precisión, no solo para determinar las mejores condiciones medioambientales de crecimiento, sino para la predicción del crecimiento mediante cálculos matemáticos

(Sharma et al.,

bloques incompletos. Se establecieron 3 bloques: (1) en la parte baja (Campo Verde - Nueva Requena), (2) media (Neshuya - Curimana), y (3) alta (San Alejandro) de la cuenca del Río Aguaytía. En cada parte de la cuenca se estableció 5 repeticiones completando 15 repeticiones para toda la cuenca. En 12 de las repeticiones supérstites (que sobrevivieron hasta los 64 meses) se calculó el índice de sitio basado en la altura promedio del quintil superior de todos los árboles (Sharma et al., 2001) a 64 meses. Se comparó los índices de sitio generados por cada una de las repeticiones y los boques; se aplicó adicionalmente una prueba de correlación para someter a prueba la influencia de las variables medio ambientales respecto al crecimiento.
2001) es por ello que ese será el parámetro propuesto para estimar las alturas dominantes a los 64 meses.

\section{Resultados y discusión. \\ Crecimiento.}

En la Tabla 1 se resume los resultados encontrados para cada una de las variables de las repeticiones en estudio. En el análisis solo se tomó en cuenta los resultados de las 12 parcelas superstites de los 3 bloques. Se presenta también el índice de sitio (IS) calculado para su comparación con los diferentes parámetros. Posteriormente se analizó cada una de las variables por separado y su

Tabla 1. Resumen de las variables de crecimiento para plantaciones de Calycophyllum spruceanum Benth. por repeticiones a los 64 meses de edad.

\begin{tabular}{|c|c|c|c|c|c|c|c|c|c|}
\hline Repetición & $\begin{array}{c}\text { Densidad } \\
\text { (individuos } \\
\text { ha }^{-1} \text { ) }\end{array}$ & $\begin{array}{c}\text { Porcentaje de } \\
\text { árboles } \\
\text { sobrevivientes } \\
\text { a los } 64 \\
\text { meses (\%) }\end{array}$ & $\begin{array}{l}\text { Dap } \\
(\mathrm{cm})\end{array}$ & $\begin{array}{l}\text { IMA Dap } \\
\left(\mathrm{cm} \cdot \text { año }^{-1}\right)\end{array}$ & $\begin{array}{c}\text { Altura } \\
\text { Total } \\
\text { (m) }\end{array}$ & $\begin{array}{c}\text { IMA } \\
\text { altura } \\
\text { Total } \\
\left(\text { m.año }^{-1}\right)\end{array}$ & $\begin{array}{l}\text { IS } \\
(\mathrm{m})\end{array}$ & $\begin{array}{c}\text { Altitud } \\
\text { aprox. } \\
\text { (m.s.n.m.) }\end{array}$ & $\begin{array}{c}\text { Extensión } \\
\text { (ha) }\end{array}$ \\
\hline $1 *$ & 1312 & 82 & 3.6 & 0.6 & 4.1 & 0.7 & 5.9 & 160 & 0.25 \\
\hline 5 & 752 & 47 & 5.6 & 0.8 & 6.1 & 1 & 8.2 & 160 & 0.25 \\
\hline 6 & 796 & 49.8 & 9.2 & 1.4 & 10 & 1.6 & 11.7 & 190 & 0.25 \\
\hline $7^{*}$ & 1236 & 77.3 & 5.6 & 0.9 & 6.1 & 1 & 8.5 & 190 & 0.25 \\
\hline 8 & 788 & 49.3 & 10.9 & 1.7 & 12.8 & 2.1 & 14.1 & 190 & 0.25 \\
\hline 9 & 792 & 49.5 & 8.6 & 1.3 & 9.9 & 1.6 & 12.4 & 190 & 0.25 \\
\hline $10 *$ & 1132 & 70.8 & 6.4 & 1 & 7 & 1.1 & 9 & 190 & 0.25 \\
\hline 11 & 800 & 50 & 12.7 & 1.9 & 13.4 & 2.2 & 14.9 & 250 & 0.25 \\
\hline 12 & 796 & 49.8 & 11.2 & 1.7 & 12.1 & 2 & 14.1 & 250 & 0.25 \\
\hline 13 & 768 & 48 & 12.3 & 1.9 & 13.2 & 2.1 & 14.9 & 250 & 0.25 \\
\hline 14 & 796 & 49.8 & 12.5 & 1.9 & 13.5 & 2.2 & 15.3 & 250 & 0.25 \\
\hline 15 & 784 & 49 & 10 & 1.5 & 10.3 & 1.7 & 11.6 & 250 & 0.25 \\
\hline
\end{tabular}


relación con el IS generado.

En la Tabla 2 se resume los resultados encontrados para cada una de las variables de los bloques en estudio. En el análisis sólo se tomó en cuenta los resultados de las 12 parcelas superstites de los 3 bloques.

Diámetro a la altura del Pecho (Dap).

En la Tabla 3 se presenta los diámetros máximos, mínimos y promedios por repetición a los 64 meses de edad por repetición, así como sus incrementos medios anuales (IMA). Es notorio destacar que el mayor crecimiento se ha observado en las parcelas ubicadas en la parte alta de la cuenca (bloque 3) donde el diámetro promedio por repetición (repetición 11) alcanza los $12.7 \mathrm{~cm}$ siendo el promedio del bloque 3 de $11.7 \mathrm{~cm}$. Los resultados menores se hallan en las zonas mas bajas de la cuenca (bloque 1) donde el diámetro promedio del bloque solo alcanza los $4.3 \mathrm{~cm}$, notándose una correspondencia directa entre la precipitación pluvial y el crecimiento. La distribución de diámetros de todas las repeticiones evaluadas muestra una distribución normal. En la zona intermedia de la cuenca se observan comportamientos muy disímiles entre sí, donde se destaca la repetición 8 con un promedio de $10.9 \mathrm{~cm}$ de DAP; se puede apreciar también en todos los casos una buena correlación entre el IS por repetición generado y el DAP por repetición (Tabla 2).

Altura total.

Calycophyllum

spruceanum es considerada una especie de mediano o rápido crecimiento, alcanzando una altura total promedio de hasta $13.5 \mathrm{~m}$ a los 64 meses en la repetición catorce perteneciente a la parte alta de la cuenca, lo que da como resultado un incremento medio anual en altura total de 2.2 m.año ${ }^{-1}$. En los resultados por bloque la altura total en el bloque 3 (parte alta de la cuenca) alcanza un promedio de 12.5 m y un IMA de 2 m.año ${ }^{-1}$ (Tabla 2). Cabe resaltar que solamente 3 de las repeticiones (1, 7 y 10) fueron raleadas según evaluación fenotípica, lo que aumenta el promedio de altura de estas repeticiones en relación con las no
Tabla 2. Resumen de las variables de crecimiento para plantaciones de Calycophyllum spruceanum Benth. por bloques a los 64 meses de edad.

\begin{tabular}{cccccccc}
\hline Bloque & $\begin{array}{c}\text { Número } \\
\text { promedio } \\
\text { de } \\
\text { árboles } \\
\text { por } \\
\text { hectárea } \\
(\mathrm{N})\end{array}$ & $\begin{array}{c}\text { Porcentaje } \\
\text { de árboles } \\
\text { actuales } \\
\text { con } \\
\text { respecto } \\
\text { al original } \\
(\%)\end{array}$ & $\begin{array}{c}\text { Dap } \\
(\mathrm{cm})\end{array}$ & $\begin{array}{c}\text { IMA } \\
\text { Dap } \\
\left(\mathrm{cm} . \mathrm{anno}^{-}\right.\end{array}$ & $\begin{array}{c}\text { Altura } \\
\text { Total } \\
(\mathrm{m})\end{array}$ & $\begin{array}{c}\text { IMA } \\
\text { altura } \\
\text { Total } \\
\left(\mathrm{m} . \text { año }^{1}\right.\end{array}$ & $\begin{array}{c}\text { IS } \\
(\mathrm{m})\end{array}$ \\
\hline 1 & 1032 & 64.5 & 4.3 & 0.8 & 4.8 & 0.9 & 7 \\
2 & 948.8 & 59.3 & 7.8 & 1.5 & 8.7 & 1.6 & 11.1 \\
3 & 788.8 & 49.3 & 11.7 & 2.2 & 12.5 & 2.3 & 14.2 \\
\hline
\end{tabular}

raleadas. El IS generado guarda una estrecha relación con la altura total, esto se apreció inclusive en las parcelas raleadas. En la Tabla 4 se presenta las alturas

Tabla 3. Incremento medio anual (IMA) de DAP y alturas totales por repetición de Calycophyllum spruceanum Benth. a los 64 meses de edad.

\begin{tabular}{|c|c|c|c|c|c|c|c|c|}
\hline & \multicolumn{8}{|c|}{ Repetición } \\
\hline & \multicolumn{2}{|c|}{1} & \multicolumn{2}{|c|}{5} & \multicolumn{2}{|c|}{6} & \multicolumn{2}{|c|}{7} \\
\hline & $\begin{array}{l}\text { Dap } \\
\text { (cm) }\end{array}$ & $\begin{array}{c}\mathrm{h} \\
(\mathrm{m})\end{array}$ & $\begin{array}{l}\text { Dap } \\
(\mathrm{cm})\end{array}$ & $\begin{array}{c}\mathrm{h} \\
(\mathrm{m})\end{array}$ & $\begin{array}{l}\text { Dap } \\
\text { (cm) }\end{array}$ & $\begin{array}{c}\mathrm{h} \\
(\mathrm{m})\end{array}$ & $\begin{array}{l}\text { Dap } \\
\text { (cm) }\end{array}$ & $\begin{array}{c}\mathrm{h} \\
(\mathrm{m})\end{array}$ \\
\hline Máximo & 8.9 & 7.5 & 10 & 11 & 14 & 15 & 11.7 & 11.1 \\
\hline Mínimo & 1 & 1.1 & 1.2 & 1.8 & 3.7 & 4.5 & 1.7 & 2.6 \\
\hline Prom. Ind. Actual & 3.6 & 4.1 & 5.6 & 6.1 & 9.2 & 10 & 5.6 & 6.1 \\
\hline \multirow[t]{4}{*}{ IMA promedio } & 0.7 & .76 & 1 & 1.1 & 1.7 & 1.9 & 1.1 & 1.1 \\
\hline & \multicolumn{8}{|c|}{ Repetición } \\
\hline & \multicolumn{2}{|c|}{8} & \multicolumn{2}{|c|}{9} & \multicolumn{2}{|c|}{10} & \multicolumn{2}{|c|}{11} \\
\hline & $\begin{array}{l}\text { Dap } \\
\text { (cm) }\end{array}$ & $\begin{array}{c}\mathrm{h} \\
(\mathrm{m})\end{array}$ & $\begin{array}{l}\text { Dap } \\
\text { (cm) }\end{array}$ & $\begin{array}{c}\mathrm{h} \\
(\mathrm{m})\end{array}$ & $\begin{array}{l}\text { Dap } \\
\text { (cm) }\end{array}$ & $\begin{array}{c}\mathrm{h} \\
(\mathrm{m})\end{array}$ & $\begin{array}{l}\text { Dap } \\
\text { (cm) }\end{array}$ & $\begin{array}{c}\mathrm{h} \\
(\mathrm{m})\end{array}$ \\
\hline Máximo & 15.4 & 22 & 14.4 & 14.9 & 13.2 & 17.1 & 18.1 & 17.2 \\
\hline Mínimo & 3.7 & 6 & 1.5 & 2.6 & 1.9 & 1.5 & 3.5 & 3.5 \\
\hline Prom. Ind. Actual & 10.9 & 12.8 & 8.6 & 10 & 6.4 & 7 & 12.7 & 13.4 \\
\hline \multirow[t]{4}{*}{ IMA promedio } & 2 & 2.4 & 1.6 & 1.9 & 1.2 & 1.3 & 2.4 & 2.5 \\
\hline & \multicolumn{8}{|c|}{ Repetición } \\
\hline & \multicolumn{2}{|c|}{12} & \multicolumn{2}{|c|}{13} & \multicolumn{2}{|c|}{14} & \multicolumn{2}{|c|}{15} \\
\hline & $\begin{array}{l}\text { Dap } \\
(\mathrm{cm})\end{array}$ & $\begin{array}{c}\mathrm{h} \\
(\mathrm{m})\end{array}$ & $\begin{array}{l}\text { Dap } \\
(\mathrm{cm})\end{array}$ & $\begin{array}{c}\mathrm{h} \\
(\mathrm{m})\end{array}$ & $\begin{array}{l}\text { Dap } \\
\text { (cm) }\end{array}$ & $\begin{array}{c}\mathrm{h} \\
(\mathrm{m})\end{array}$ & $\begin{array}{l}\text { Dap } \\
\text { (cm) }\end{array}$ & $\begin{array}{c}\mathrm{h} \\
(\mathrm{m})\end{array}$ \\
\hline Máximo & 16 & 17 & 19 & 17.5 & 19.4 & 17.8 & 15.8 & 13.3 \\
\hline Mínimo & 4.3 & 4.9 & 3 & 2.5 & 2.9 & 1.7 & 2.8 & 2 \\
\hline Prom. Ind. Actual & 11.2 & 12.1 & 12.3 & 13.2 & 12.5 & 13.5 & 10 & 10.3 \\
\hline IMA promedio & 2.1 & 2.3 & 2.3 & 2.5 & 2.3 & 2.5 & 1.9 & 1.9 \\
\hline
\end{tabular}

Tabla 4. Incrementos Medios Anuales (IMA) de Dap y alturas totales por bloques Calycophyllum spruceanum Benth. a los 64 meses de edad.

\begin{tabular}{|c|c|c|c|c|c|c|}
\hline & \multicolumn{6}{|c|}{ Bloque } \\
\hline & \multicolumn{2}{|c|}{1} & \multicolumn{2}{|c|}{2} & \multicolumn{2}{|c|}{3} \\
\hline & $\begin{array}{l}\text { Dap } \\
(\mathrm{cm})\end{array}$ & $\begin{array}{c}\mathrm{H} \\
(\mathrm{m})\end{array}$ & $\begin{array}{l}\text { Dap } \\
(\mathrm{cm})\end{array}$ & $\begin{array}{c}\mathrm{H} \\
(\mathrm{m})\end{array}$ & $\begin{array}{l}\text { Dap } \\
(\mathrm{cm})\end{array}$ & $\begin{array}{c}\mathrm{h} \\
(\mathrm{m})\end{array}$ \\
\hline Máximo & 10 & 11 & 15.4 & 17.1 & 19.4 & 17.8 \\
\hline Mínimo & 1 & 1.1 & 1.5 & 1.5 & 2.8 & 1.7 \\
\hline $\begin{array}{c}\text { Promedio Individual } \\
\text { Actual }\end{array}$ & 4.3 & 4.8 & 7.8 & 8.7 & 11.7 & 12.5 \\
\hline IMA promedio & 0.8 & 0.9 & 1.5 & 1.6 & 2.2 & 2.3 \\
\hline
\end{tabular}


Tabla 5. Resumen de productividad por repeticiones para Calycophyllum spruceanum Benth. a los 64 meses de edad.

\begin{tabular}{ccccc}
\hline Repetición & $\begin{array}{c}\text { Area } \\
\text { basal } \\
\left(\mathrm{m}^{2} \mathrm{ha}^{-1}\right)\end{array}$ & $\begin{array}{c}\text { Volumen } \\
\left(\mathrm{m}^{3} \mathrm{ha}^{-1}\right)\end{array}$ & $\begin{array}{c}\text { IMA Volumen } \\
\left(\mathrm{m}^{3} \mathrm{ha}^{-1} \mathrm{ano}^{-1}\right)\end{array}$ & $\begin{array}{c}\text { IS } \\
(\mathrm{m})\end{array}$ \\
\hline $1 *$ & 1.6 & 5 & 0.936 & 5.9 \\
5 & 2 & 8.5 & 1.598 & 8.2 \\
6 & 5.5 & 35.4 & 6.641 & 11.7 \\
$7 *$ & 3.4 & 14.6 & 2.745 & 8.5 \\
8 & 7.6 & 60.9 & 11.409 & 14.1 \\
9 & 5 & 32.8 & 6.154 & 12.4 \\
$10^{*}$ & 4 & 19.2 & 3.593 & 9 \\
11 & 10.5 & 87.5 & 16.41 & 14.9 \\
12 & 8.2 & 62.2 & 11.671 & 14.1 \\
13 & 9.6 & 80.3 & 15.046 & 14.9 \\
14 & 10.1 & 85.1 & 15.965 & 15.3 \\
15 & 6.3 & 40.6 & 7.612 & 11.6 \\
\hline * Parcela raleada & & &
\end{tabular}

Tabla 6. Resumen de productividad por bloques de las plantaciones de Calycophyllum spruceanum Benth. a los 64 meses de edad.

\begin{tabular}{ccccc}
\hline Bloque & $\begin{array}{c}\text { Área basal } \\
\left(\mathrm{m}^{2} \mathrm{ha} \mathrm{a}^{-1}\right)\end{array}$ & $\begin{array}{c}\text { Volumen } \\
\left(\mathrm{m}^{3} \mathrm{ha}^{-1}\right)\end{array}$ & $\begin{array}{c}\text { IMA Volumen } \\
\left(\mathrm{m}^{3} \mathrm{ha}^{-1} \mathrm{anoo}^{-1}\right)\end{array}$ & $\begin{array}{c}\text { IS } \\
(\mathrm{m})\end{array}$ \\
\hline 1 & 1.76 & 6.279 & 1.177 & 7 \\
2 & 4.88 & 29.915 & 5.609 & 11.1 \\
3 & 8.95 & 71.196 & 13.349 & 14.2 \\
\hline
\end{tabular}

máximas, mínimas y promedios a los 64 meses de edad por repetición, así como sus incrementos medios anuales (IMA), alcanzando hasta 2.5 m.año ${ }^{-1}$ en la mejor repetición (11). También se presentan los IMA de alturas por bloques donde el incremento alcanza hasta 2.3 m.año ${ }^{-1}$. Estos datos son muy superiores a los reportados en Brasil para rodales naturales y parcelas experimentales de zona no aluvial (Oliveira, 2000) pero inferiores a los reportados para zona aluvial (Courturier, 1994; Sears, 2003).

Área basal y volumen.

La Tabla 5 presenta el resumen de área basal y volumen de acuerdo a los bloques, alcanzando 8.54 $\mathrm{m}^{2} \mathrm{ha}^{-1}$ en el promedio del bloque 3 (parte alta de la cuenca), notándose también una alta correspondencia entre el área basal y el IS calculado para todos los bloques. La repetición alcanza el mayor valor con 10 $\mathrm{m}^{2} \mathrm{ha}^{-1}$ en la repetición 11. Cabe destacar que las repeticiones raleadas presentan menor área basal por la menor cantidad de árboles, y que los menores índices de área basal se encuentran en las repeticiones del bloque 1 (parte baja de la cuenca). En todos los casos se aprecia una alta correspondencia entre los valores del área basal y el índice de sitio calculado para todas las repeticiones.

En la Tabla 6 se muestra el área basal por bloques alcanzando $8.54 \mathrm{~m}^{2} \mathrm{ha}^{-1}$ en el promedio del bloque 3 (parte alta de la cuenca), notándose también una alta correspondencia entre el área basal y el IS calculado para todos los bloques.

Volumen.

El cálculo de volumen fue realizado según lo reportado para la especie en esta misma zona para volúmenes con corteza a partir de un DAP y la altura total correspondiente con un factor de forma de 0.58 (Pinedo, 2004). La Tabla 5 describe los resultados del volumen por repetición alcanzando en la repetición 11 un volumen de $81.572 \mathrm{~m}^{3} \mathrm{ha}^{-1}$ y un IMA volumétrico de $15.295 \mathrm{~m}^{3} \mathrm{ha}^{-1}$. La Tabla 6 describe los resultados por bloques, encontrándose una gran variabilidad entre los bloques a los 64 meses, pues el rango se encuentra entre 4.397 y $63.990 \mathrm{~m}^{3} \mathrm{ha}^{-1}$. Sin embargo el IMA volumétrico por bloque es inferior al reportado por repetición alcanzando los $11.998 \mathrm{~m}^{3} \mathrm{ha}^{-1}$ lo que sin embargo se considera un nivel de productividad entre medio y alto para especies de densidad alta, similar al reportado para otras especies en la zona en diferentes sistemas de plantación (Flores, 2002). Una revisión de crecimientos anuales y densidades por especie reportada en plantaciones forestales en América tropical se presenta en el Anexo 1 para fines comparativos.

Diámetros y alturas del quintil superior.

En la Tabla 7 se presentan los incrementos medios anuales de quintil superior por repetición, lo que da una buena aproximación del estado del desarrollo de las repeticiones en condiciones optimas de supervivencia y crecimiento, siendo el máximo Dap individual de $19.4 \mathrm{~cm}$ (repetición 14) con un IMA promedio por repetición en diámetro de $2.9 \mathrm{~cm}$ (repetición 14). En referencia al promedio por repetición de altura total, este alcanza $15.5 \mathrm{~m}$ (repetición 11, 13 y 14), siendo la altura total máxima por repetición de $17.81 \mathrm{~m}$ (repetición 14) siendo el IMA de altura promedio mas alto $2.8 \mathrm{~m}$ (repetición 14), en este caso los árboles evaluados coinciden con aquellos seleccionados para calcular el índice de sitio.

En la Tabla 8 se presentan los incrementos medios anuales de quintil superior por bloque, siendo notoria la superioridad del bloque 3 con $2.7 \mathrm{~cm}$ de IMA diámetro y 2.7 m de IMA de altura.

Índice de sitio.

En la Tabla 9 se muestran las correlaciones entre el índice de sitio y los parámetros establecidos: Altura total (h) Diámetro a la altura del pecho (Dap), Área Basal y Volumen promedio por repetición, todas las correlaciones halladas son altamente significativas inclusive en las parcelas no raleadas, lo cual es coherente con lo reportado en la literatura. La correlación más alta es con la altura total promedio (0.99) esta correlación va descendiendo para los otros parámetros Dap (0.97), Área basal (0.92) y finalmente Volumen (0.9), estableciéndose que el índice de sitio es adecuado para proyectar en forma rápida $y$ económica el potencial crecimiento de una plantación. 
Una proyección preliminar de la curva de los índices de sitio se presenta en el anexo 4.

En cuanto a la correlación de parámetros de evaluación (Tabla 10) por bloque las correlaciones son más altas debido principalmente al mayor número de individuos, así tenemos la altura (0.99), Dap (0.98) y Volumen (0.95), que por tratarse de datos experimentales biométricos son altamente significativas.

Variables de climáticas e índice de sitio.

La Figura 3 presenta gráficamente el Dap, Área Basal y Volumen por repetición donde se aprecia claramente que hay dos notables excepciones a las tendencias de los bloques: la repetición 8 que presenta estas tres variables notablemente superior a todas las repeticiones del bloque 2 pues se encuentra en la zona de influencia aluvial y la repetición 15 que tiene un promedio de crecimiento notablemente inferior a las demás repeticiones del bloque 3, en ambos casos la distribución de los bloques coincide con las isoyetas de precipitación, por lo que las excepciones mencionadas no se explican por variables climáticas, ni por condiciones edáficas ya que existen diferencias mínimas en los valores de suelos, tampoco de micrositio, sino probablemente por ataques de plagas y enfermedades, la parcela 15 tiene un porcentaje muy bajo de árboles sin ataques, lo que coincide con los bajos rendimientos de las parcelas 7 y 10 (ver anexo 2).

\section{Conclusiones.}

En plantaciones puras a 5.3 años de edad Calycophyllum spruceanum obtuvo un crecimiento de hasta $12.7 \mathrm{~cm}$ en DAP, un incremento medio anual (IMADAP) de $2.4 \mathrm{~cm}$, $13.37 \mathrm{~m}$ de altura total, $\mathrm{y}$ un incremento medio anual de altura (IMAH) de $2.507 \mathrm{~m}, 10.17 \mathrm{~m} 2$ de área basal y $81.572 \mathrm{~m}^{3} /$ ha de volumen. Estos resultados fueron notablemente más altos que aquellos ubicados en la zona baja de la cuenca y consistentes con una distribución de sitio, siendo las plantaciones más productivas aquellas que se ubican en la parte más alta de la cuenca del Aguaytía, con mayores precipitaciones.

La evaluación mediante el índice de sitio, definido como el quintil superior de árboles con mayor altura, ha sido en este caso eficiente para representar las variables de crecimiento de las plantaciones como DAP promedio, altura promedio, área basal y volumen.

Los resultados preliminares de crecimiento de plantaciones puras Calycophyllum spruceanum sugieren fomentar este sistema de plantación en zonas de precipitación alta y media (mayores de $2500 \mathrm{~mm} \mathrm{año}^{-1}$ ), con suelos aluviales, ph de ácido a
Tabla 7. Incrementos Medios Anuales (IMA) de DAP y alturas totales del quintil superior de las repeticiones de Calycophyllum spruceanum Benth. a los 64 meses de edad.

\begin{tabular}{|c|c|c|c|c|c|c|c|c|}
\hline & \multicolumn{8}{|c|}{ Repetición } \\
\hline & \multicolumn{2}{|c|}{$1^{*}$} & \multicolumn{2}{|c|}{5} & \multicolumn{2}{|c|}{6} & \multicolumn{2}{|c|}{ 7* } \\
\hline & $\begin{array}{l}\text { Dap } \\
(\mathrm{cm})\end{array}$ & $\begin{array}{c}\mathrm{h} \\
(\mathrm{m})\end{array}$ & $\begin{array}{l}\text { Dap } \\
\text { (cm) }\end{array}$ & $\begin{array}{c}\mathrm{h} \\
(\mathrm{m})\end{array}$ & $\begin{array}{l}\text { Dap } \\
(\mathrm{cm})\end{array}$ & $\begin{array}{l}\mathrm{h} \\
(\mathrm{m})\end{array}$ & $\begin{array}{l}\text { Dap } \\
(\mathrm{cm})\end{array}$ & $\begin{array}{c}\mathrm{h} \\
(\mathrm{m})\end{array}$ \\
\hline Máximo & 7 & 7.5 & 10 & 11 & 13.2 & $\begin{array}{c}13 . \\
8\end{array}$ & 11.7 & 11.1 \\
\hline Mínimo & 5 & 4.4 & 7 & 6. & 10.9 & 9.3 & 7.4 & 3.8 \\
\hline $\begin{array}{c}\text { Promedio } \\
\text { Individual } \\
\text { Actual }\end{array}$ & 5.7 & 5.9 & 8 & 8.2 & 11.7 & $\begin{array}{c}11 . \\
7\end{array}$ & 8.4 & 8.5 \\
\hline $\begin{array}{c}\text { IMA } \\
\text { promedio }\end{array}$ & 1.1 & 1.1 & 1.5 & 1.5 & 2.2 & 22 & 1.6 & 1.6 \\
\hline & \multicolumn{8}{|c|}{ Repetición } \\
\hline & \multicolumn{2}{|c|}{8} & \multicolumn{2}{|c|}{9} & \multicolumn{2}{|c|}{10} & \multicolumn{2}{|c|}{11} \\
\hline & $\begin{array}{l}\text { Dap } \\
(\mathrm{cm})\end{array}$ & $\begin{array}{c}\mathrm{h} \\
(\mathrm{m})\end{array}$ & $\begin{array}{l}\text { Dap } \\
(\mathrm{cm})\end{array}$ & $\begin{array}{c}\mathrm{h} \\
(\mathrm{m})\end{array}$ & $\begin{array}{l}\text { Dap } \\
\text { (cm) }\end{array}$ & $\begin{array}{c}\mathrm{h} \\
(\mathrm{m})\end{array}$ & $\begin{array}{l}\text { Dap } \\
\text { (cm) }\end{array}$ & $\begin{array}{c}\mathrm{h} \\
(\mathrm{m})\end{array}$ \\
\hline Máximo & 15.4 & $\begin{array}{c}16 . \\
5\end{array}$ & 14.4 & 14.9 & 13.2 & $\begin{array}{c}17 . \\
1\end{array}$ & 18.1 & 16.7 \\
\hline Mínimo & 12.5 & $\begin{array}{c}11 . \\
1\end{array}$ & 10.7 & 10.3 & 8.1 & 6.9 & 14.6 & 12.6 \\
\hline $\begin{array}{c}\text { Promedio } \\
\text { Individual } \\
\text { Actual }\end{array}$ & 13.5 & $\begin{array}{l}14 . \\
11\end{array}$ & 11.7 & 12.4 & 9.2 & 9 & 15.5 & 14.9 \\
\hline \multirow[t]{4}{*}{$\begin{array}{c}\text { IMA } \\
\text { promedio }\end{array}$} & 2.5 & 2.6 & 2.2 & 2.3 & 1.7 & $\begin{array}{c}16 . \\
9\end{array}$ & 2.9 & 2.8 \\
\hline & \multicolumn{8}{|c|}{ Repetición } \\
\hline & \multicolumn{2}{|c|}{12} & \multicolumn{2}{|c|}{13} & \multicolumn{2}{|c|}{14} & \multicolumn{2}{|c|}{15} \\
\hline & $\begin{array}{l}\text { Dap } \\
(\mathrm{cm})\end{array}$ & $\begin{array}{c}\mathrm{h} \\
(\mathrm{m})\end{array}$ & $\begin{array}{l}\text { Dap } \\
(\mathrm{cm})\end{array}$ & $\begin{array}{c}\mathrm{h} \\
(\mathrm{m})\end{array}$ & $\begin{array}{l}\text { Dap } \\
(\mathrm{cm})\end{array}$ & $\begin{array}{l}\mathrm{h} \\
(\mathrm{m})\end{array}$ & $\begin{array}{l}\text { Dap } \\
(\mathrm{cm})\end{array}$ & $\begin{array}{c}\mathrm{h} \\
(\mathrm{m})\end{array}$ \\
\hline Máximo & 16 & 17 & 19 & 17.5 & 19.4 & $\begin{array}{c}17 . \\
8\end{array}$ & 15.8 & 13.3 \\
\hline Mínimo & 12.9 & 12 & 14.7 & 11.1 & 14.4 & $\begin{array}{c}10 . \\
9\end{array}$ & 11.2 & 5.8 \\
\hline $\begin{array}{c}\text { Promedio } \\
\text { Individual } \\
\text { Actual }\end{array}$ & 13.8 & $\begin{array}{c}14 . \\
1\end{array}$ & 15.5 & 14.9 & 15.5 & $\begin{array}{c}15 . \\
3\end{array}$ & 12.3 & 11.6 \\
\hline $\begin{array}{c}\text { IMA } \\
\text { promedio }\end{array}$ & 2.6 & 2.6 & 2.9 & 2.8 & 2.9 & 2.9 & 2.3 & 2.2 \\
\hline
\end{tabular}

* No Raleado

Tabla 8. Incrementos Medios Anuales (IMA) de DAP y alturas totales del quintil superior por bloques de Calycophyllum spruceanum Benth. a los 64 meses de edad.

\begin{tabular}{ccccccc}
\hline & \multicolumn{9}{c}{ Bloque } \\
\hline & \multicolumn{2}{c}{1} & \multicolumn{2}{c}{2} & \multicolumn{2}{c}{3} \\
\cline { 2 - 7 } & $\begin{array}{c}\text { Dap } \\
(\mathrm{cm})\end{array}$ & $\begin{array}{c}h \\
(\mathrm{~m})\end{array}$ & $\begin{array}{c}\text { Dap } \\
(\mathrm{cm})\end{array}$ & $\begin{array}{c}h \\
(\mathrm{~cm})\end{array}$ & $\begin{array}{c}\text { Dap } \\
(\mathrm{cm})\end{array}$ & $\begin{array}{c}h \\
(\mathrm{~cm})\end{array}$ \\
\hline Máximo & 10 & 11 & 15.4 & 17.1 & 19.4 & 17.8 \\
$\begin{array}{c}\text { Mínimo } \\
\text { Promedio }\end{array}$ & 5 & 4.4 & 7.4 & 3.8 & 11.2 & 5.8 \\
$\begin{array}{c}\text { Individual } \\
\text { Actual } \\
\text { IMA }\end{array}$ & 6.9 & 0.7 & 11 & 11.1 & 14.5 & 14.2 \\
promedio & 1.3 & 1.3 & 2.1 & 2.1 & 2.7 & 26.6 \\
\hline
\end{tabular}


Tabla 9. Relación del índice de Sitio (IS) con la Altura total, DAP, Área basal y Volumen promedio por repetición de Calycophyllum spruceanum Benth. a los 64 meses de edad.

\begin{tabular}{cccccc}
\hline REPETICIÓN & $\begin{array}{c}\text { IS } \\
(\mathrm{m})\end{array}$ & $\begin{array}{c}\mathrm{h} \\
(\mathrm{m})\end{array}$ & $\begin{array}{c}\text { Dap } \\
(\mathrm{cm})\end{array}$ & $\begin{array}{c}\mathrm{AB} \\
(\mathrm{m} 2 / \mathrm{ha})\end{array}$ & $\begin{array}{c}\text { Volúmen } \\
(\mathrm{m} 3)\end{array}$ \\
\hline $1^{*}$ & 5.9 & 4.2 & 1.6 & 4.99 & 3.321 \\
5 & 8.2 & 6.1 & 2.0 & 8.52 & 6.676 \\
6 & 11.7 & 10 & 5.5 & 35.42 & 31.337 \\
$7 *$ & 8.5 & 6.1 & 3.4 & 14.64 & 11.154 \\
8 & 14.1 & 12.8 & 7.6 & 60.85 & 56.301 \\
9 & 12.4 & 9.9 & 5 & 32.82 & 27.311 \\
$10^{*}$ & 9 & 7 & 4.0 & 19.16 & 15.284 \\
11 & 14.9 & 13.4 & 10.5 & 87.52 & 81.572 \\
12 & 14.1 & 12.1 & 8.2 & 62.24 & 57.331 \\
13 & 14.9 & 13.2 & 9.6 & 80.25 & 72.358 \\
14 & 15.3 & 13.5 & 10.1 & 85.14 & 78.264 \\
15 & 11.6 & 10.3 & 6.3 & 40.6 & 37.991 \\
Correlación & 0.99 & 0.97 & 0.92 & 0.9 \\
\hline *Parcela raleada & & & & &
\end{tabular}

ligeramente ácido, baja saturación de aluminio. Para impulsar un programa adecuado del repoblamiento comercial de esta especie, la decisión de establecer las plantaciones debe considerar un análisis económico referente a costos y rendimientos de las operaciones forestales, desde el establecimiento hasta la transformación primaria.

Debido a las características de crecimiento de la especie se recomienda seguir con el registro de crecimiento y desarrollo hasta alcanzar el primer turno de corta que debe tomar según las proyecciones entre 16 y 20 años. Eventualmente se deberían probar adicionalmente las características físicas y mecánicas de las maderas procedentes de plantaciones para considerar esta variable en el cálculo de la proyección de edad de cosecha. Se debería también integrar en la evaluación de las plantaciones el índice de sitio como un parámetro de gran exactitud para la evaluación de las variables silviculturales como el volumen, por su gran practicidad y representatividad para las estimaciones en plantaciones.

Tabla 10. Relación del Índice de Sitio (IS) con la Altura total, DAP, Área basal y volumen promedio por bloque de Calycophyllum spruceanum Benth. a los 64 meses de edad.

\begin{tabular}{|c|c|c|c|c|c|}
\hline Bloque & $\begin{array}{l}\text { IS } \\
(\mathrm{m})\end{array}$ & $\begin{array}{c}\mathrm{h} \\
(\mathrm{m})\end{array}$ & $\begin{array}{l}\text { Dap } \\
(\mathrm{cm})\end{array}$ & $\begin{array}{c}\mathrm{AB} \\
\left(\mathrm{m}^{2} \mathrm{ha}^{-1}\right)\end{array}$ & $\begin{array}{c}\text { Volúmen } \\
\left(\mathrm{m}^{3}\right)\end{array}$ \\
\hline 1 & 7 & 4.8 & 4.3 & 1.76 & 6.279 \\
\hline 2 & 11.1 & 8.7 & 7.8 & 4.88 & 29.915 \\
\hline 3 & 14.2 & 12.5 & 11.7 & 8.95 & 71.196 \\
\hline \multicolumn{2}{|c|}{ Correlación } & 0.9934 & 0.9836 & 0.9816 & 0.9533 \\
\hline
\end{tabular}

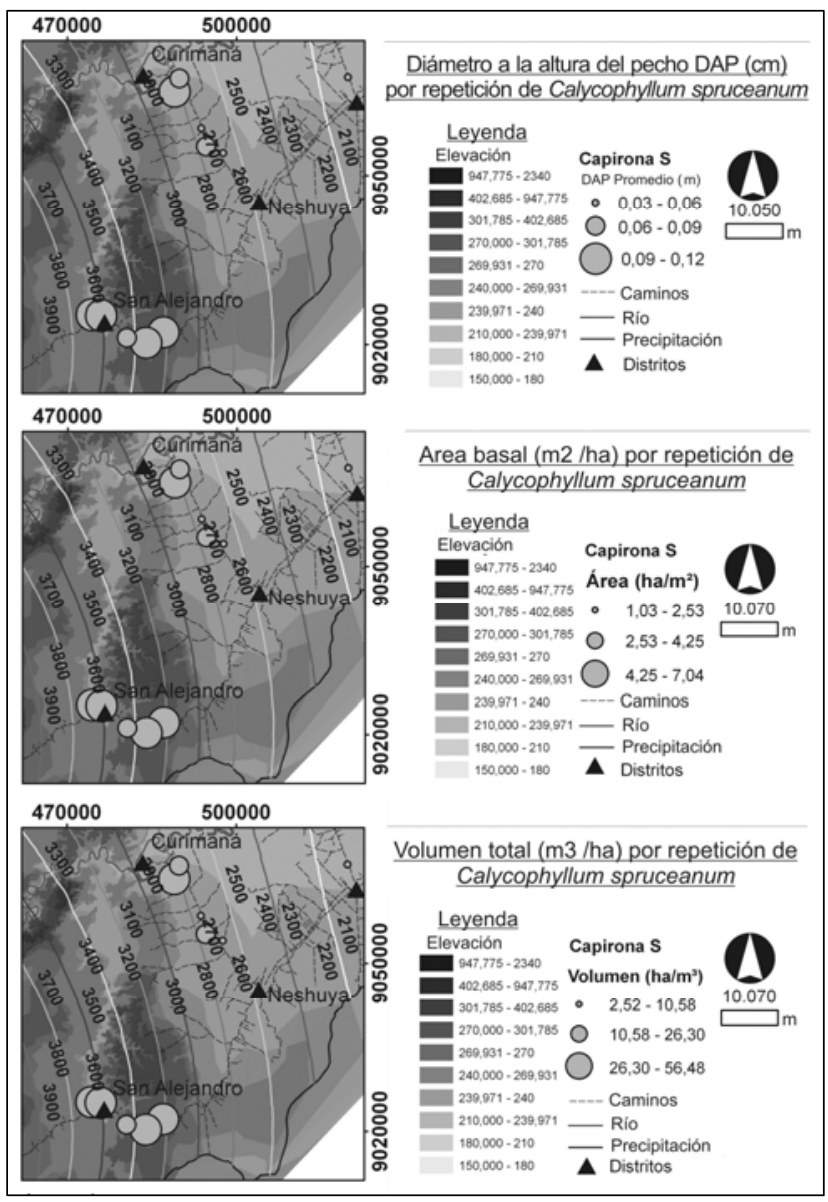

Figura 3. Dap, Área basal y Volumen por repetición de Callycophyllum spruceanum Benth. en la cuenca del río Aguaytía, Ucayali, Perú.

\section{Agradecimientos.}

A la Dora Samaniego y Zoila Vela por la edición del manuscrito.

\section{Literatura citada.}

Armitage F. \& Burley J. 1980. Pinus kesiya en: Tropical forestry paper $\mathrm{N}^{\circ}$ 9. Department of forestry, commonwealth forestry Institute. University of Oxford. England. Reino Unido.

Avery T. \& Bukhart H. 1994. Forest measurements. Nueva York, EUA 408 p.

Baskaran D. 2001. Regional report south and east Asia. FAO panel of experts on forest genetic resources twelfth session Rome 21 -23. Noviembre 2001, Italy.

Beck D. 1971. Polymorphic site index curves for white pine in the Southern Appalachian en: Research Paper SE-80. United State Department of Agriculture, Forest Services.

Caillez F. \& Alder D. 1980. Forest Volume Estimation and Yield Production. Vol. I. Forest Volume Estimation. FAO Forestry Paper n ${ }^{\circ}$ 22/1. Roma.

Canadian Forester Services. 2002. The state of Canada's Forest 2001-2002.

Carmean W. 1971. Site Index Curves for Black, White, Scarlet, And Chestnut Oaks In The Central States, en: 
USDA Forest Service. Research Paper NC-62. St. Paul, Minesota, EUA.

Casaubon E., Gurini L. \& Cueto G. 2000. Relaciones entre características estacionales y la productividad en una plantación de Salís nigra 4 en el delta Bonaerense del Río Paraná (Argentina). Investigación Agraria: Sistemas y recursos forestales. 29(2). Buenos Aires, Argentina.

Courturier G. \& Gonzales J. 1994. Insectos plaga de la capirona Calycophyllum spruceanum Benth (Rubiaceae), en sistema agroforestal. Revista Peruana de Entomología 37:97-99.

Chapman H. \& Myer W. 1949. Forest Mensuration. Nueva York USA.

Daniels T., Helms J. \& Baker F. 1979. Principles of Silviculture. Nueva York, EUA.

Flores Y. 2002. Crecimiento y productividad de plantaciones de seis especies forestales nativas de 20 años de edad en el bosque Alexander Von Humboldt, Amazonía Peruana. Tesis para optar al grado de Magister Scientiae, Centro agronómico tropical de Investigación e Enseñanza CATIE, Turrialba, Costa Rica.

Flores Y. 2008. Ecuación de índice de sitio para Cedrelinga catenaeformis en el Bosque Alexander von Humboldt.

Froese K., LeMay V., Marshall P. \& Zumrawi A. 2002. Small Tree Height Increment Models for Prognosis ${ }^{\mathrm{BC}}$, IDFdm2 Subzone Variant, Invermere Forest District. Report prepared for: Forestry Innovation Investment, Reference No: R02-07. Forest Resources Management Department, University of British Columbia.

García O. 1999. Hieight growth of Pinus radiata in New Zealand. New Zealand Journal of Forestry Sciences. 29(1): 131-145.

Hanson E. Azuma D. \& Hirerote B. 2002. Site Index Equations and Mean Annual Increment Equations for Pacific Northwest Research Station Forest Inventory y Analysis Inventories, 1985-2001. USDA Forest Service. Research Note PNW-RN-533. Portland, Oregon, EUA.

Hijmans R.J., Cameron S.E., Parra J.L., Jones P.G. \& Jarvis A. 2005. Very high resolution interpolated climate surfaces for global land areas. International Journal of Climatology 25: 1965-1978.

Holdridge L. 1979. Ecología basada en zonas de vida. Serie de Libros y Materiales Educativos, San José, Costa Rica.

Huebschmann M. \& Martin J. 1996. Estimating and Interpreting Site Index. Wisconsin USA.

Jones R. 1969. Review and comparison of site evaluation methods en: Forest research paper R-M 51 United State Department of Agriculture USDA.

Lencar C. \& Marshall P. 2000. Small Tree Height Growth and stocking in the IDF dk1, dk2 and dk3 subzone, Kamloops and Cariboo Forest Regions. Report to the Forest Practices and Research Branches B. C. Ministry of Forests. Forest Resources Management Department. University of British Columbia.

McNab W. \& Ritter B. 1999. Site index evaluation in a 100 year old eastern white pine plantation at the biltmore estate, NC en: Tenth Biennial Southern Silvicultural Research Conferences, Shreveport. LA, February 1999.

Oliveira M. 2000. Artificial regeneration gaps and skidding trails after mechanized forest exploitation in Acre Brazil in: Forest ecology and management 127: 67-76.

Parker R., Matney T. \& Belli K. 2003. Field and Laboratory Exercises for Forest Description and Analysis. Edition 7. Department of forestry, College of forestry Resources. Missisippi State University: $130-133$.

Pinedo A. 2004. Tabla de volumen de Calycophyllum spruceanum en la cuenca del río Aguaytía, Tesis para optar al grado de ingeniero forestal, Universidad Nacional de Ucayali, Pucallpa, Perú.

Prodan M., Peters R., Cox F. \& Real P. 1997. Mensura Forestal. San José, Costa Rica.

Sears R. 2003. New Forestry on The Floodplain: The Ecology And Management of Calycophyllum spruceanum (Rubiacea) on the Amazon Landscape. Tesis de Doctorado. Universidad de Columbia. Nueva York, EUA.

Sharma M., Amateis R. \& Burkhart H. 2001. Top height definition and its effect on site index determination in thinned and unthinned loblolly pine plantations. Forest Ecology and Management 168(1-3): 163-175.

Sims D. 1994. Southern Hardwood, Hardwood Site and Stand Inventory Management en: Management Bulletin R8-MB 67. Cooperative extension service, The University of Georgia.

Sotelo C. \& Weber J. 2001. Variation in growth and wood traits among provenances of Calycophyllum spruceanum Benth. from the Peruvian Amazon. Research Report International Tropical Timber Organization Scholarship Programme Reference 090/99A. Pucallpa, Perú.

Thrower J. 1989. Site Quality Evaluation Using Site Index. Training Course, Module III, Silviculture Institute of British Columbia. Surrey, British Columbia.

Torres-Rojo, J. 2001. Curvas de índice de sitio de forma y escala variable en investigación forestal. Agrociencia 35: 87-98

Tyler A., MacMillan D. \& Dutch J. 1996. Models to predict the general yield class of Douglas fir, Japanese larch and Scots pine on better quality land in Scotland. Forestry. 69(1): 13 - 24.

Wykoff W. 1990. A basal Area Icrement Model for Individual Conifers in the Northern Rocky Mountains in: For. Sci. 36(4): 1077 - 1104.

Zedaker S.M., Lewis J.B. Smith D.WM. \& Kreh R.E. 1987. Impact of season of harvest and site quality on cutstump treatments of Piedmont hardwoods. Southern Journal of Applied Forestry 11: 46-49. 
Anexo 1. Tabla comparativa de Incremento medios anuales de Volumen $\left(\mathrm{m}^{3} \mathrm{ha}^{-1} \mathrm{año}^{-1}\right)$ y densidades básicas $\left(\mathrm{gr}^{1} \mathrm{~cm}^{-3}\right)$.

\begin{tabular}{|c|c|c|c|}
\hline Especie & $\begin{array}{l}\text { Densidad } \\
\mathrm{g} / \mathrm{cm} 3\end{array}$ & $\begin{array}{l}\text { Crecimiento } \\
\mathrm{m} 3 / \mathrm{ha} / \mathrm{año}\end{array}$ & Fuente \\
\hline Acacia magnum & 0.50 & 39 & $\begin{array}{l}\text { OLIVA-HURTARTE, E. } 1990 . \text { Tesis, Mag. Sc, Centro } \\
\text { Agronómico Tropical de Investigación y Enseñanza, Turrialba } \\
\text { (Costa Rica). Comportamiento en plantación de mangium } \\
\text { (Acacia mangium Willd) y aripin (Caesalpinia velutina (B y R) } \\
\text { Standl) en América Central. }\end{array}$ \\
\hline Amburana cearensis & 0.50 & 3.27 & $\begin{array}{l}\text { FLORES, Y et al. 2004. Crecimiento y productividad de } \\
\text { plantaciones de seis especies forestales nativas de } \\
20 \text { años de edad en el bosque Alexander Von Humboldt, } \\
\text { Amazonia Peruana. }\end{array}$ \\
\hline Cedrela odorata & 0.38 & $\begin{array}{l}12.6 \\
\mathrm{a} \\
11.7\end{array}$ & $\begin{array}{l}\text { LAMB. 1996. Mahogany of tropical America. Its ecology and } \\
\text { management. Univ Michigan. }\end{array}$ \\
\hline Cedrelinga catenaeformis & 0.44 & 21.43 & $\begin{array}{l}\text { FLORES, Y et al. 2004. Crecimiento y productividad de } \\
\text { plantaciones de seis especies forestales nativas de } \\
20 \text { años de edad en el bosque Alexander Von Humboldt, } \\
\text { Amazonia Peruana. }\end{array}$ \\
\hline Cedrelinga catenaeformis & 0.45 & 44.6 & RICSE, A. 2006.INFORME DE GESTIÓN \\
\hline Cedrelinga cateniformis & 0.45 & 23 & $\begin{array}{l}\text { ALDER, D. 1999. Growth and yield of some plantation species } \\
\text { of the lowland tropics in Ecuador. }\end{array}$ \\
\hline Copaifera reticulata & 0.6 & 3.3 & $\begin{array}{l}\text { FLORES, Y et al. 2004. Crecimiento y productividad de } \\
\text { plantaciones de seis especies forestales nativas de } \\
20 \text { años de edad en el bosque Alexander Von Humboldt, } \\
\text { Amazonia Peruana. }\end{array}$ \\
\hline Cordia alliodora & 0.55 & $20-25$ & $\begin{array}{l}\text { RIZZO, P. Especies seleccionas para la forestación: eucalipto } \\
\text { tropical, teca, melina, pino caribe, guayacán, laurel, balsa, } \\
\text { eucalipto glóbulos y pino. }\end{array}$ \\
\hline Eucalyptus grandis & $\begin{array}{l}0.40 \\
\mathrm{a} \\
0.59\end{array}$ & $\begin{array}{l}49.8 \\
59, \\
56.6 \\
64.9 \text { y } \\
62 .\end{array}$ & $\begin{array}{l}\text { ENDO, M. 1994. Aumento del crecimiento debido al control de } \\
\text { la maleza en una plantación de E. grandis de cuatro años de edad } \\
\text { en Colombia. Resultados del primer año. }\end{array}$ \\
\hline Gmelina arborea, & $\begin{array}{l}0.40 \\
\mathrm{a} \\
0.58\end{array}$ & 30 & $\begin{array}{l}\text { RIZZO, P. Especies seleccionas para la forestación: eucalipto } \\
\text { tropical, teca, melina, pino caribe, guayacán, laurel, balsa, } \\
\text { eucalipto glóbulos y pino. }\end{array}$ \\
\hline Jacaranda copaia & 0.31 & 16 & $\begin{array}{l}\text { ALDER, D. 1999. Growth and yield of some plantation species } \\
\text { of the lowland tropics in Ecuador. }\end{array}$ \\
\hline Pino oregon & 0.38 & 27 & $\begin{array}{l}\text { HONORATO. 2002. Evaluación de las forestaciones del Campo } \\
\text { Experimental Trevelin Ing. Ftal. Luis Tejera - Téc. Univ. Ftal. }\end{array}$ \\
\hline Pinus caribaea & 0.48 & $20-25$ & $\begin{array}{l}\text { RIZZO, P. Especies seleccionas para la forestación: eucalipto } \\
\text { tropical, teca, melina, pino caribe, guayacán, laurel, balsa, } \\
\text { eucalipto glóbulos y pino. }\end{array}$ \\
\hline Pinus radiata & 0.39 & 17.7 & $\begin{array}{l}\text { GONZALEZ, M. Crecimiento de Pinus radiata en Cajamarca } \\
\text { Perú }\end{array}$ \\
\hline Schizolobium parahybum & 0.3 & 10 a 23 & $\begin{array}{l}\text { ALDER, D. 1999. Growth and yield of some plantation species } \\
\text { of the lowland tropics in Ecuador. }\end{array}$ \\
\hline Simarouba amara & 0.36 & 6.8 & $\begin{array}{l}\text { FLORES, Y. et al. 2004. Crecimiento y productividad de } \\
\text { plantaciones de seis especies forestales nativas de } 20 \text { años de } \\
\text { edad en el bosque Alexander Von Humboldt, Amazonia Peruana. }\end{array}$ \\
\hline Tectona grandis & $\begin{array}{l}0.53 \\
\mathrm{a} \\
0.574\end{array}$ & $10.2-13.3$ & $\begin{array}{l}\text { PEREZ CORDERO, et al 2000. Desarrollo de escenarios de } \\
\text { crecimiento para plantaciones de teca (Tectona grandis) en Costa } \\
\text { Rica. }\end{array}$ \\
\hline
\end{tabular}


Anexo 2. Diagramas climáticos de la zona de estudio.

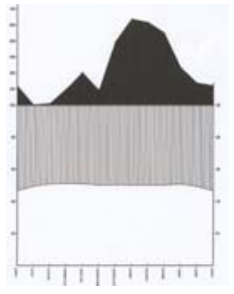

Aguaytía

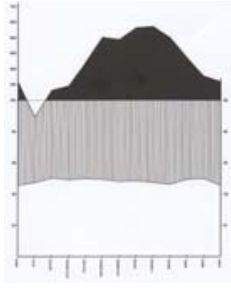

San Alejandro

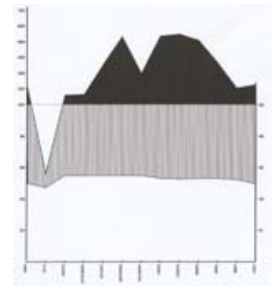

Von Humboldt

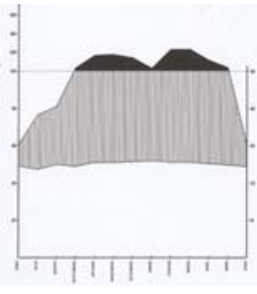

San Jorge

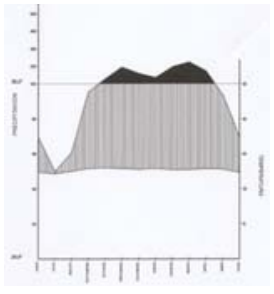

Pucallpa

Anexo 3. Porcentaje (\%) de individuos respecto a su Estado Sanitario con respecto al número total de ejes vivos por repeticiones en plantaciones puras de Calycophyllum spruceanum

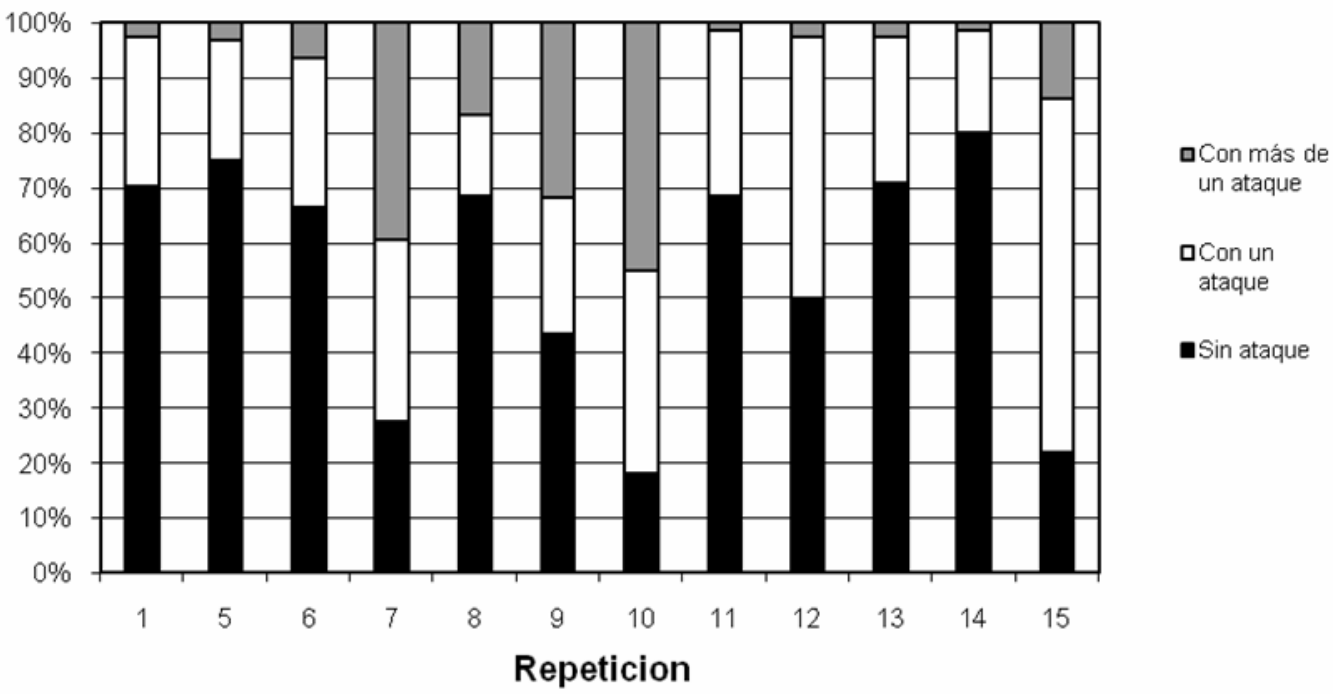

Anexo 4. Curva de índice de sitio Calycophyllum spruceanum Benth. a una edad clave de 5 años $\left(\mathrm{IS}_{5}\right)$ en Ucayali, Perú.

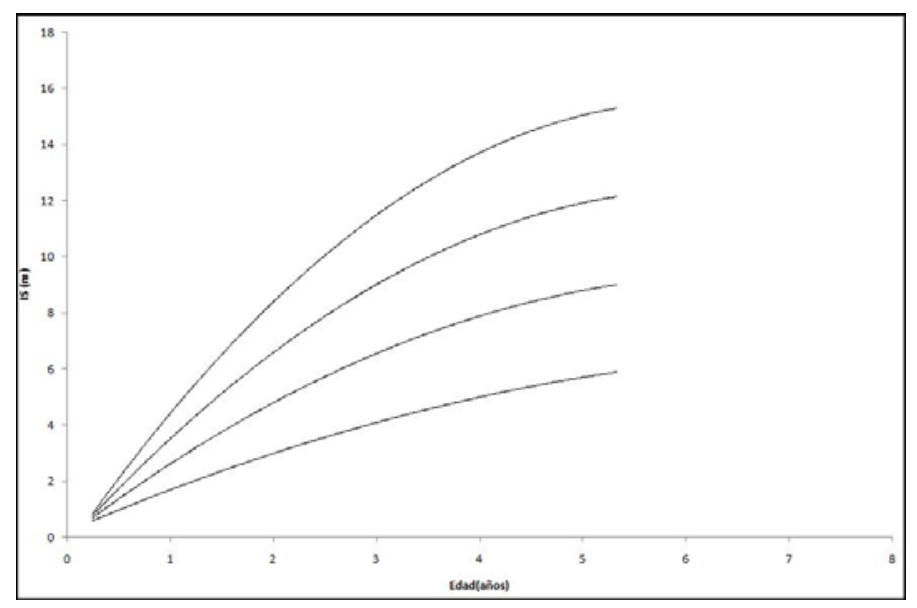

\footnotetext{
${ }^{1}$ World Agroforestry Center (ICRAF). jugarte@cgiar.org.

${ }^{2}$ Profesor Principal UNALM. gdominguez@lamolina.edu.pe.
} 